Tropical Journal of Pharmaceutical Research October 2021; 20 (10): 2135-2142

ISSN: $1596-5996$ (print); 1596-9827 (electronic) (C) Pharmacotherapy Group, Faculty of Pharmacy, University of Benin, Benin City, 300001 Nigeria

\title{
Impact of citalopram combined with mindfulness-based stress reduction on symptoms, cognitive functions and self-confidence in patients with depression
}

\author{
Zhenyu Chen ${ }^{1}$, Langyun Yan², Xiaoyu Nong ${ }^{3}$, Fuguang Chen ${ }^{1}$, Fengkun Gan ${ }^{4}$, \\ Peizhi Tang ${ }^{5}$, Jinmei Qin ${ }^{6}$, Yingmin Huang ${ }^{7 *}$, Li Huang ${ }^{8}$ \\ ${ }^{1}$ College of Teacher Education, Xichang University, Xichang, China, ${ }^{2}$ Wuzhou No. 3 Middle School, Wuzhou, 3 Basic Teaching \\ Department, College of Guangxi Industrial and Commercial Technician, Wuzhou, China., ${ }^{4}$ Department of Pediatrics, Yulin \\ Hospital of Traditional Chinese Medicine, Yulin, ${ }^{5}$ College of Education Science, Guangxi Science \& Technology Normal \\ University, Nanning, ${ }^{6}$ Psychological Consultation Center, Guangxi Transport Vocational and Technical College, Nanning, \\ ${ }^{7}$ Department of Clinical Psychology, Yulin Fourth People's Hospital, Yulin, ${ }^{8}$ Psychological Consultation Center, Guangxi \\ Technical School of News Publication, Nanning, China
}

*For correspondence: Email: 823213599@qq.com; greataids@163.com Tel: +86-15177615370

\begin{abstract}
Purpose: To investigate the impact of the combination of citalopram and mindfulness-based stress reduction (MBSR) on the symptoms, cognitive functions and self-confidence of patients with depression. Methods: A total of 98 patients with depression were selected as study subjects and divided into combination therapy group $(C T, n=51)$ and conventional group $(C, n=47$. The conventional group was treated with citalopram, while the combined group was treated with a combination of citalopram and MBSR. Depressive symptoms and self-confidence were evaluated using the 17-item Hamilton Depression Rating Scale (HAMD-17) and General Self-efficacy Scale (GSES). Cognitive functions were assessed by Wisconsin Card Sorting Test (WCST) and Trail Making Test (TMT). Changes in depressive symptoms, cognitive functions, self-confidence and clinical efficacies between the two groups were compared.

Results: At weeks 1, 4 and 8 after treatment, CT group had lower HAMD-17 scores but higher GSES scores when compared with the conventional group $(p<0.05)$. In addition, CT group was superior to the conventional group in efficacy and overall response rate (100.00 vs. $85.11 \%, p<0.05)$. Also, CT group showed a shorter time of perseverative and non-perseverative errors on WCST and a shorter time for $T M T-A$ and TMT-B, compared with the conventional group $(p<0.05)$.

Conclusion: The combination therapy of citalopram and MBSR is effective in ameliorating depressive symptoms, and enhancing cognitive functions and self-confidence in patients with depression. These findings will increase the understanding of this combination therapy, and provide a clinical reference for the treatment of depression.
\end{abstract}

Keywords: Citalopram, Mindfulness-based Stress Reduction, Depression, Cognitive functions, Selfconfidence

This is an Open Access article that uses a funding model which does not charge readers or their institutions for access and distributed under the terms of the Creative Commons Attribution License (http://creativecommons.org/licenses/by/4.0) and the Budapest Open Access Initiative (http://www.budapestopenaccessinitiative.org/read), which permit unrestricted use, distribution, and reproduction in any medium, provided the original work is properly credited.

Tropical Journal of Pharmaceutical Research is indexed by Science Citation Index (SciSearch), Scopus, International Pharmaceutical Abstract, Chemical Abstracts, Embase, Index Copernicus, EBSCO, African Index Medicus, JournalSeek, Journal Citation Reports/Science Edition, Directory of Open Access Journals (DOAJ), African Journal Online, Bioline International, Open-J-Gate and Pharmacy Abstracts 


\section{INTRODUCTION}

Depression is a common mental disorder induced by genetic factors, mediator dysfunction in the central nervous system, constitutional factors, and mental factors. The clinical symptoms of depression are mainly, persistent and obvious depression, delusions, hallucinations, as well as suicidal thoughts and behavior in severe cases. Depression is known to have a high prevalence due to increased recurrence rates, and as such frequent incidences become chronic, the resultant effect is an elevated difficulty in treatment and rehabilitation. A study has suggested that the incidence rate of major depression in China exceeds $3.5 \%$ [1]. Most patients suffering from depression display signs of cognitive impairment, leading to a lack of the self-confidence needed to overcome the disease, and consequently an inability to restore their social functions as soon as possible [2].

Currently, medication is primarily adopted for the treatment of depression. Although medication can improve the health of patients already suffering from clinical symptoms of depression such as delusions and hallucinations, as well as control the progression of depression, most patients tend to show a high recurrence rate of depression, a low cure rate, as well as impaired cognitive functions. A study indicated that medication demonstrates an efficacy of appropriately $67 \%$ in the treatment of depression, and the residual depressive symptoms lead to impaired cognitive functions and a dip in the patient's self-confidence, thus negatively affecting the efficacy of the medication [3]. Patients with depression cannot be cured using medication alone. Medication combined with psychotherapy remarkably improves the efficacy of medication, improves patients' cognitive functions, and their ability to regain their self-confidence to overcome it, as well as reduce residual depressive symptoms [4].

Citalopram, which is known to alleviate depressive symptoms, is a commonly used firstline drug for the treatment of depression. However, it remains unclear whether citalopram improves cognitive function. Mindfulness-based Stress Reduction (MBSR), also known as mindfulness meditation, is an eight-week evidence-based program that offers secular, intensive mindfulness training (e.g., body scan, mindful breathing, meditation as well as walking meditation) to improve patients' consciousness, concentration and emotions. It has been reported that MBSR is highly convenient and effective [5]. Previous evidence indicates that MBSR effectively relieves physical and psychological stress, as well as stress-induced anxiety and depression [6]. Mindfulness-based stress reduction can be implemented in a healthy and sub-healthy population, as well as patients with chronic diseases, cancer and mental illness. This study explores the impact of citalopram combined with MBSR on symptoms, cognitive function and self-confidence in patients with depression, so as to provide a reference for the treatment of depression.

\section{METHODS}

\section{Patients}

A total of 98 patients with depression were selected as the study subjects. Inclusion criteria: 1) the 17-item Hamilton Depression Rating Scale (HAMD-17) score $\geq 7$ points, and diagnosed as suffering from depression by an experienced chief physician in psychiatry. Exclusion criteria: 1) inability to complete the investigation (e.g., denial of anxiety, absentmindedness, dysgnosia); 2) allergic to the study drugs; 3) drug abuser or alcoholics; 4) high suicidal tendency; 5) preconception, pregnancy or lactation. The patients were divided into combined group $(\mathrm{n}=$ 51) and conventional group ( $n=47)$, in accordance with different therapeutic methods. This study was approved by the Ethics Committee of Yulin Fourth People's Hospital (approval no. YLFH202009020003). The research subjects and their families were informed, and they signed a fully-informed consent form. The study was conducted in compliance with the guidelines of the Declaration of Helsinki.

\section{Treatments}

The conventional group was treated with $10 \mathrm{mg}$ of citalopram per day for 8 weeks (SFDA approval No::H20080786, Enterprise Name: Sichuan Kelun Pharmaceutical Co. Ltd, Chengdu, China). Depending on the actual conditions of the patients in this group, the maximum dose administered was 15 - $20 \mathrm{mg}$.

The combined group on the other hand, was treated with MBSR, and nursing intervention was carried out in accordance with the mindfulness theory five times a week (30-45 min for each session) for 8 weeks. A long-term, comfortable and serene location was selected, and after the activity was concluded, homework and related materials were assigned to patients for implementation (Table 1). 
Table 1: Main contents of MBSR

\begin{tabular}{|c|c|}
\hline Time & Content \\
\hline Week 1 & $\begin{array}{l}\text { Frequent communications with patients were conducted to establish a sound nurse-patient relationship, } \\
\text { gain patients' trust, and understand patients' strong and weak points, personalities and individual } \\
\text { preferences, the patients were informed of the causes of depression, the adverse effects of negative } \\
\text { emotions on their diseases, and the measures to improve the symptoms were learned. Knowledge and } \\
\text { concepts related to mindfulness were introduced using raisin exercises, the patients were instructed to } \\
\text { perceive raisins through feeling, smell and vision, and to perceive the changes in body, feeling, and } \\
\text { emotions. }\end{array}$ \\
\hline Week 2 & $\begin{array}{l}\text { Body scan was learned by the patients, and the patients were instructed to close their eyes to perceive } \\
\text { the sensations in different parts of the body and the connection among different parts of the body. Then, } \\
\text { the patients conducted mindful breathing, took comfortable sitting positions, closed their eyes, slowly } \\
\text { exhaled from their noses, slowly bulged their abdomens, slowly exhaled from their mouths, and then } \\
\text { slowly dropped their abdomen. Next, the patients performed the mindfulness meditation, perceived } \\
\text { changes in the body, listened to the surrounding sounds, and perceived the emergence, development and } \\
\text { disappearance of ideas at the time of mindful breathing and abdominal ups and downs. }\end{array}$ \\
\hline Week 3 & $\begin{array}{l}\text { The patients conducted the walking meditation, devoted the attention to the contiguous sense between } \\
\text { the soles of the feet and the ground when surnamed Zhou, raised their feet, moved, put the feet down, } \\
\text { walked slowly to the end, and then walked to the starting point. }\end{array}$ \\
\hline Week 4-7 & $\begin{array}{l}\text { The comprehensive exercises (i.e., body scan, mindful breathing, mindfulness meditation, and walking } \\
\text { meditation) were performed with a focus on the perception of every motion, and the analysis and } \\
\text { judgment were not conducted. }\end{array}$ \\
\hline Week 8 & $\begin{array}{l}\text { The patients were instructed to learn to adjust their depressive emotions and conduct things that make } \\
\text { them happy, so as to have positive emotions. The patients continued conducting the exercises that had } \\
\text { been performed previously, conducted the summarization, and provided feedbacks. Meanwhile, the } \\
\text { patients were encouraged to implement everything they have learned in their lives, and accept their } \\
\text { depressive symptoms, so as to reduce their negative emotions. }\end{array}$ \\
\hline
\end{tabular}

\section{Observational indexes}

Prior to the commencement of treatment, and at weeks 1, 4 and 8 after treatment, depressive symptoms were evaluated using HAMD-17 [7]. The HAMD-17 comprises 17 items. Some items were scored based on 0 - 4 points, while some were scored based on 0-2 points. A higher HAMD-17 score indicated a more serious depressive symptom. Evaluation criteria for efficacy [8]: At Week 8 after treatment, HAMD-17 recorded a score of 7 points or $95 \%$ reduction compared with the score before treatment, thus indicating cured; HAMD-17 scored 7 points, representing a $70 \%-95 \%$ reduction compared with before treatment, indicated a marked response; a HAMD-17 score of 7 points or $0 \%$ $69 \%$ reduction compared with before treatment, indicating a moderate response; and finally a HAMD-17 score of $50 \%$ reduction compared with before treatment, indicating no response. Before treatment commenced and then 8 weeks after treatment, the cognitive functions were evaluated using the Wisconsin Card Sorting Test (WCST) [9] and the Trail Making Test (TMT) [10]. WCST was run using a computer, and according to the number, shape and color randomly displayed by the computer, the subjects were classified, and the number of perseverative and nonperseverative errors, as well as the number of classified subjects were recorded. TMT was divided into TMT-A and TMT-B. In TMT-A, 25 Arabic numerals were randomly distributed on a white paper (size: 16k), and subjects were required to connect them in sequence. In TMT-B, 1-13 Arabic numerals and letters $A-L$ were also randomly distributed on a white paper (size: 16k), and subjects were required to alternately connect Arabic numerals and letters in sequence. The time for connection was recorded. Finally, selfconfidence was evaluated before treatment and at weeks 1, 4 and 8 after treatment, using the General Self-efficacy Scale (GSES). GSES consists of 10 items [11], and each item was scored based on a range of 1 - 4 points. A higher GSES score indicated a higher sense of selfefficacy.

\section{Statistical analysis}

All data were processed using Statistic Package for Social Science (SPSS) 22.0 (IBM, Armonk, NY, USA). Enumeration data are expressed as $(\mathrm{n}, \%)$, and detected using $X^{2}$ test. Measurement data are expressed as (mean \pm SD). The intragroup comparison was carried out using and independent sample t-test, while the intergroup comparison was implemented using the paired $t-$ test. The comparisons at different time points were performed using analysis of variance (ANOVA) of repeatedly measured data, so as to analyze the differences between the groups and the time differences of the values measured at the various time points. Subsequently, the least significant difference (LSD) t-test was performed. 
$P<0.05$ indicated statistically significant difference.

\section{RESULTS}

\section{Baseline data}

There was no difference in the baseline data (i.e., age, gender, course of disease and educational level between the two groups $(p>$ 0.05, Table 2).

\section{Depressive symptoms}

Repeated measurements showed that there were marked differences in HAMD-17 scores between the two groups at different time points, and the interactions of time points between the groups were significantly different $(p<0.05)$. Subsequently, LSD-t revealed that the comparison between the HAMD-17 scores of the two groups suggests that HAMD-17 scores at week 8 after treatment, HAMD-17 scores at week 4 after treatment, HAMD-17 scores at week 1 after treatment, HAMD-17 scores before treatment, and the HAMD-17 scores in the combined group were notably lower than those in the conventional group at week 1, 4 and 8 after treatment $(p<0.05$; Table 3 and Figure 1).

\section{Efficacy of treatment}

The combined group was superior to the conventional group in efficacy and overall response rate (100.00 vs. $85.11 \% ; p<0.05$, Table 4).

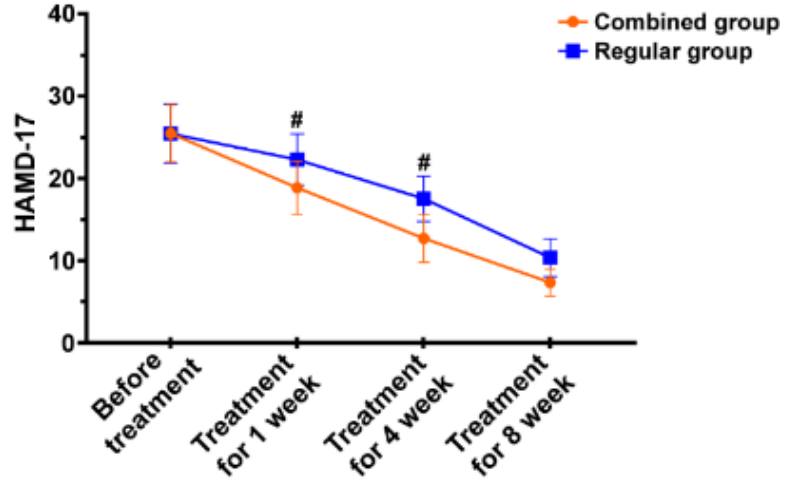

Figure 1: Comparison of the HAMD-17 scores between the two groups at different time points. There is no obvious difference in HAMD-17 scores between the two groups before treatment $(p>0.05)$, and the HAMD-17 scores in the combined group are notably lower than those in the conventional group at weeks 1 , 4 and 8 after treatment comparison between the two groups at the same time points

\section{Cognitive functions}

Before treatment, there was no significant difference in the time of perseverative and nonperseverative errors on WCST, the number of classified subjects, and the time of TMT-A and TMT-B between the two groups $(p>0.05)$. After treatment, the time of perseverative and nonperseverative errors on WCST and the time duration of TMT-A and TMT-B in the two groups were shortened compared with those before treatment $(p<0.05)$.

Table 2: Comparison of baseline data between the two groups (mean $\pm S D, n, \%$ )

\begin{tabular}{|c|c|c|c|c|c|c|c|}
\hline \multirow[t]{2}{*}{ Group } & \multirow[t]{2}{*}{ Age (yr) } & \multicolumn{2}{|c|}{ Gender } & \multirow{2}{*}{$\begin{array}{l}\text { Course of disease } \\
\text { (months) }\end{array}$} & \multicolumn{3}{|c|}{ Educational level } \\
\hline & & Male & Female & & $\begin{array}{l}\text { Junior high school } \\
\text { and below }\end{array}$ & $\begin{array}{l}\text { High } \\
\text { school }\end{array}$ & $\begin{array}{c}\text { Junior college and } \\
\text { above }\end{array}$ \\
\hline $\begin{array}{l}\text { Combined } \\
(51)\end{array}$ & $\begin{array}{c}39.47 \pm 8 \\
.25\end{array}$ & $\begin{array}{c}14 \\
(27.45)\end{array}$ & $\begin{array}{c}37 \\
(72.55)\end{array}$ & $4.52 \pm 1.42$ & $10(19.61)$ & $\begin{array}{c}15 \\
(29.41)\end{array}$ & $26(50.98)$ \\
\hline $\begin{array}{l}\text { Conventional } \\
(47)\end{array}$ & $\begin{array}{l}40.12 \pm 8 \\
.03\end{array}$ & $\begin{array}{c}15 \\
(31.91)\end{array}$ & $\begin{array}{c}32 \\
(68.09)\end{array}$ & $4.98 \pm 1.49$ & $9(19.15)$ & $\begin{array}{c}16 \\
(34.04)\end{array}$ & $22(46.81)$ \\
\hline$t$ & 0.395 & & 234 & 1.565 & & 0.106 & \\
\hline$P$-value & 0.694 & 0. & 629 & 0.121 & & 0.745 & \\
\hline
\end{tabular}

Table 3: Comparison of HAMD-17 scores between the two groups at different time points $(n, \%)$

\begin{tabular}{|c|c|c|c|c|c|}
\hline Group & $\begin{array}{c}\text { Number of } \\
\text { cases }\end{array}$ & $\begin{array}{c}\text { Before } \\
\text { treatment }\end{array}$ & $\begin{array}{c}\text { Week } 1 \text { after } \\
\text { treatment }\end{array}$ & $\begin{array}{c}\text { Week } 4 \text { after } \\
\text { treatment }\end{array}$ & Week 8 after treatment \\
\hline Combined & 51 & $25.49 \pm 3.48$ & $18.86 \pm 3.24^{\text {ad }}$ & $12.71 \pm 2.90^{\mathrm{abd}}$ & $7.35 \pm 1.65^{\mathrm{abcd}}$ \\
\hline Conventional & 47 & $25.45 \pm 3.60$ & $22.28 \pm 3.13^{a}$ & $17.53 \pm 2.74^{\mathrm{ab}}$ & $10.36 \pm 2.31^{\mathrm{abc}}$ \\
\hline$F$ & & \multicolumn{4}{|c|}{$F_{\text {time points }}=1120.092, F_{\text {interaction }}=16.992, F_{\text {between groups }}=37.312$} \\
\hline$P$-value & & \multicolumn{4}{|c|}{$p_{\text {time points }}=0.000<0.001, p_{\text {interaction }}=0.000<0.001, p_{\text {between groups }}=0.000<0.001$} \\
\hline
\end{tabular}


Table 4: Comparison of efficacies between the two groups at week 8 after treatment $(n, \%)$

\begin{tabular}{lcccccc}
\hline Group & $\begin{array}{c}\text { Number of } \\
\text { cases }\end{array}$ & Cured & $\begin{array}{c}\text { Marked } \\
\text { response }\end{array}$ & $\begin{array}{c}\text { Moderate } \\
\text { response }\end{array}$ & No response & ORR \\
\hline Combined & 51 & $22(43.14)$ & $9(17.65)$ & $20(39.22)$ & $0(0)$ & $51(100)$ \\
Conventional & 47 & $0(0)$ & $4(8.51)$ & $36(76.6)$ & $7(14.89)$ & $40(85.11)$ \\
$Z / X^{2}$ & & & & 30.324 & & 8.18 \\
$P$-value & & & $<0.001$ & 0.004 \\
\hline
\end{tabular}

The aforementioned time in the combined group was obviously shorter than that in the conventional group $(p<0.05)$, the number of classified subjects in the two groups remarkably increased compared with those before treatment $(p<0.05)$, and the aforementioned number in the combined group was significantly higher than that in the conventional group $(p<0.05)$ (Table $5)$.

\section{Self-confidence}

Repeated measurements demonstrated that there were significant differences in GSES scores between the groups at certain time points, and the interactions of these time points and between groups were significantly different $(p<$ 0.05 ). Subsequently, LSD-t revealed that the comparison of GSES scores between the two groups exhibited: GSES scores at week 8 after treatment > GSES scores at week 4 after treatment > GSES scores at week 1 after treatment> GSES scores before treatment; and the GSES scores in the combined group were remarkably higher than those in the conventional group ( $p<0.05$; Table 6 and Figure 2).

\section{DISCUSSION}

Mindfulness, a positive psychological quality requiring no judgment, evolves from a Buddhist practice, and it is a state of perfusion and awareness of current stimuli, and a state of consciousness that fully accepts one's own thoughts, feelings, emotions and external things without judgment, doubt or rejection [12].

Table 5: Comparison of cognitive function scores between the two groups before and after treatment (mean \pm $\mathrm{SD})$

\begin{tabular}{|c|c|c|c|c|c|c|}
\hline \multirow[t]{2}{*}{ Group } & \multirow[t]{2}{*}{ Time point } & \multicolumn{3}{|c|}{ WCST } & \multicolumn{2}{|c|}{ TMT } \\
\hline & & $\begin{array}{c}\text { Number of } \\
\text { perseverative } \\
\text { errors }\end{array}$ & $\begin{array}{l}\text { Number of non- } \\
\text { perseverative } \\
\text { errors }\end{array}$ & $\begin{array}{c}\text { Number of } \\
\text { classified subjects } \\
\text { (persons) }\end{array}$ & $\begin{array}{l}\text { A Time } \\
\text { (min) }\end{array}$ & B Time (min) \\
\hline \multirow[t]{2}{*}{ Combined (51) } & Before treatment & $26.56 \pm 8.02$ & $14.34 \pm 4.19$ & $4.89 \pm 1.19$ & $73.52 \pm 21.17$ & $119.83 \pm 35.5$ \\
\hline & After treatment & $18.48 \pm 6.17$ & $10.22 \pm 3.16$ & $7.01 \pm 0.97$ & $52.46 \pm 15.54$ & $75.29 \pm 24.43$ \\
\hline ta value & & 5.703 & 5.606 & 9.861 & 5.727 & 7.381 \\
\hline$p_{a}$ value & & $<0.001$ & $<0.001$ & $<0.001$ & $<0.001$ & $<0.001$ \\
\hline Conventional & Before treatment & $27.23 \pm 8.65$ & $15.26 \pm 4.36$ & $4.79 \pm 1.24$ & $72.59 \pm 23.24$ & $120.81 \pm 37.23$ \\
\hline group $(n=47)$ & After treatment & $21.63 \pm 6.87$ & $12.48 \pm 3.82$ & $5.89 \pm 0.88$ & $63.51 \pm 18.59$ & $87.59 \pm 25.91$ \\
\hline$t_{\mathrm{a}}$ & & 3.476 & 3.288 & 4.96 & 2.092 & 5.021 \\
\hline$p_{a}$ & & 0.001 & 0.001 & $<0.001$ & 0.039 & $<0.001$ \\
\hline$t_{\mathrm{b}}$ & & 0.398 & 1.065 & 0.407 & 0.207 & 0.133 \\
\hline$p_{\mathrm{b}}$ & & 0.692 & 0.29 & 0.685 & 0.836 & 0.894 \\
\hline$t_{c}$ & & 2.391 & 3.201 & 5.969 & 3.202 & 2.419 \\
\hline$P$-value ${ }^{c}$ & & 0.019 & 0.002 & $<0.001$ & 0.002 & 0.017 \\
\hline
\end{tabular}

Note: $\mathrm{t}_{\mathrm{a}}$ and $\mathrm{Pa}$ indicate the comparison within groups, $\mathrm{t}_{\mathrm{b}}$ and $\mathrm{P}_{\mathrm{b}}$ indicate the comparison before treatment, and $\mathrm{t}_{\mathrm{c}}$ and $\mathrm{P}_{\mathrm{c}}$ indicate the comparison after treatment

Table 6: Comparison of GSES scores between the two groups at different time points (mean \pm SD)

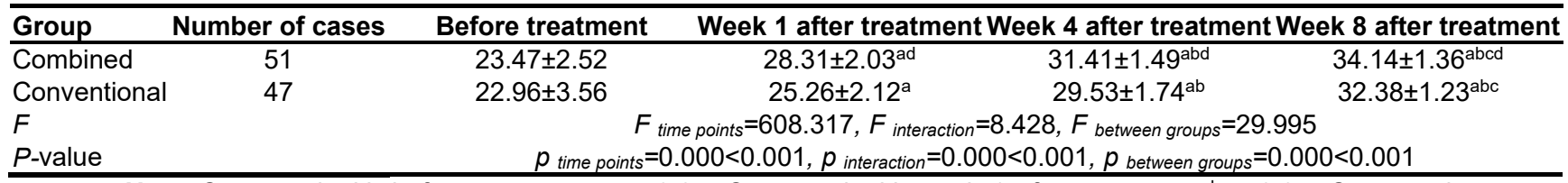

Note: Compared with before treatment, ${ }^{\mathrm{a}} p<0.05$; Compared with week 1 after treatment, ${ }^{\mathrm{b}} p<0.05$; Compared with week 4 after treatment, ${ }^{\mathrm{c}} p<0.05$; Compared with conventional group at the same time points, ${ }^{\mathrm{d}} p<0.05$ 


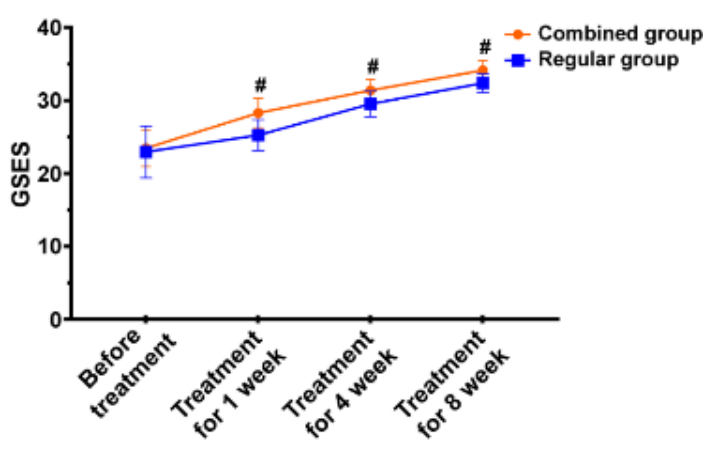

Figure 2: Comparison of GSES scores between the two groups at different time points. There is no marked difference in GSES scores between the two groups before treatment $(p>0.05)$, and the GSES scores in the combined group are remarkably higher than those in the conventional group at weeks 1,4 and 8 after treatment comparison between the two groups at the same time points

The MBSR 8-week program, first developed by Dr. Jon Kabat-Zinn in 1979, combines western psychology and eastern Buddhist meditation, and proposes skills based on mindfulness-related knowledge and ideas, so as to alleviate patients' stress, pain, anxiety, and immunity [13].

Most negative emotions such as, anger, regret, and worries are caused by past memories and future perceptions, and this results in the ignorance of the present. With a full focus on the present, less attention can be devoted to the future and the past, and thus become less plagued by adverse emotions. The core content of MBSR lies in focusing on perceiving the present. Through the perceiving emotions, thoughts and feelings and integrating them into life, the encephalic region in the left cerebral prefrontal lobe can be enhanced and activated, so that patients can remain calm and goodhumored in the face of stress, thereby effectively reducing negative emotions [14].

In this study, HAMD-17 scores in the combined group were significantly lower than those in the conventional group at week 1, 4 and 8 after treatment. The combined group was superior to the conventional group in efficacy and overall response rate $(100.00$ vs. $85.11 \%)$. The study results demonstrated that citalopram combined with MBSR could effectively improve the depressive symptoms and efficacy. This is because MBSR focuses on feelings and improves negative emotions through physical urination, mindful breathing and mindfulness meditation, which can help patients to stay in good shape and treat others with tolerance. Walking meditation can also help to release negative emotions, perceive the real-time changes, avoid perfectionism, accept individual imperfections, and make a significant contribution to preventing depressive symptoms and a relapse [15]. The results of this study revealed that the GSES scores in the combined group were noticeably higher than those in the conventional group at weeks 1,4 and 8 weeks after treatment, suggesting that citalopram combined with MBSR improved self-confidence in patients with depression. Citalopram combined with MBSR can effectively mitigate patients' depressive symptoms so as to boost patients' self-confidence in overcoming depression.

Cognitive dysfunction in patients with depression is characterized by cerebral dysfunction, negatively affecting the cognitive flexibility and concept formation. Wisconsin Card Sorting Test (WCST), a satisfactory tool useful for testing the sensitivity of cognitive functions, can be used to evaluate the concept formation and transformation of learning and memory abilities, the ability to correct mistakes, as well as the assessment of cognitive flexibility and abstract classification [16]. Trail Making Test (TMT), primarily adopted to evaluate the coordination, thinking and spatial perception of the eyes and hands of the subjects, can reflect the abilities of cognitive set shift, visual spatial ordering and rapid visual search [17].

The prefrontal lobe affects the coordination between the executive function and attention, and executive function is positively correlated with prefrontal cortex blood flow. The damaged prefrontal lobe leads to a fall in the body's awareness of the environment and its autonomy, resulting in dysgnosia [18]. Therefore, TMT can be used to evaluate whether there is prefrontal lobe dysfunction. In this study, the combined group had a shorter time of perseverative and non-perseverative errors on WCST, and a shorter time for TMT-A and TMT-B when compared with the conventional group. The study results exhibit that citalopram combined with MBSR effectively improved cognitive dysfunction in patients with depression.

\section{Limitations of the study}

However, the limitations of this study lie in the absence of long-term follow-up after treatment, non-analysis of the long-term efficacy of citalopram combined with MBSR on patients with depression, and the fact that there was no attempt to classify and explore patients with depression of different genders and age ranges, and the relatively small sample size. Therefore, future studies with a larger sample size should 
be performed to conduct long-term follow-up on patients with depression of based on gender and age range.

\section{CONCLUSION}

The combination of citalopram and MBSR can effectively mitigate depressive symptoms, and improve cognitive functions and self-confidence in patients with depression. The findings will enhance current understanding of the effect of the combination of citalopram and MBSR, and provide a clinical reference for the treatment of depression.

\section{DECLARATIONS}

\section{Acknowledgement}

This study was supported by the Foundation of Research on Technology and Quality Standards of Mixture Formula for Regulating Stomach and Releasing Excessive Turbid (no. ZRQN1509, 030029031 to Wei Jin).

\section{Conflict of Interest}

No conflict of interest associated with this work.

\section{Contribution of Authors}

The authors declare that this work was done by the authors named in this article and all liabilities pertaining to claims relating to the content of this article will be borne by them. Li Huang should be considered as the co-corresponding author.

\section{Open Access}

This is an Open Access article that uses a funding model which does not charge readers or their institutions for access and distributed under the terms of the Creative Commons Attribution License (http://creativecommons.org/licenses/by/ 4.0) and the Budapest Open Access Initiative (http://www.budapestopenaccessinitiative.org/rea d), which permit unrestricted use, distribution, and reproduction in any medium, provided the original work is properly credited.

\section{REFERENCES}

1. Lamers $F$, Milaneschi $Y$, Smit JH, Schoevers RA, Wittenberg G, Penninx B. Longitudinal Association Between Depression and Inflammatory Markers: Results From the Netherlands Study of Depression and Anxiety. Biol Psychiatry 2019; 85(10): 829-837.
2. Winter G, Hart RA, Charlesworth R, Sharpley CF. Gut microbiome and depression: what we know and what we need to know. Rev Neurosci 2018; 29(6): 629-643.

3. Garbutt J, Sterkel R, Ruecker K, Dodd S, Smith E, Plax K. Ready for the Challenge of Depression Care in the Medical Home. Clin Pediatr (Phila) 2019; 58(7): 816818.

4. Clunne SJ, Ryan BJ, Hill AJ, Brandenburg C, Kneebone I. Accessibility and Applicability of Currently Available eMental Health Programs for Depression for People With Poststroke Aphasia: Scoping Review. J Med Internet Res 2018; 20(12): e291.

5. Lengacher $C A$, Reich RR, Ramesar $S$, Alinat CB, Moscoso M, Cousin L, Marino VR, Elias MN, Paterson $C L$, Pleasant $M L$, et al. Feasibility of the mobile mindfulness-based stress reduction for breast cancer (mMBSR(BC)) program for symptom improvement among breast cancer survivors. Psychooncology 2018; 27(2): 524-531.

6. Rose SA, Sheffield D, Harling $M$. The Integration of the Workable Range Model into a Mindfulness-Based Stress Reduction Course: a Practice-Based Case Study. Mindfulness (N Y) 2018; 9(2): 430-440.

7. Shen $H$, Tu $X$, Luan $X$, Zeng $Y, H e ~ J$, Tang $W$. Serum lipid profiles and post-stroke depression in acute ischemic stroke patients. Neuropsychiatr Dis Treat 2019; 15: 1573-1583.

8. Zhao B, Li Z, Wang Y, Ma X, Wang X, Wang X, Liu J, Huang $Y$, Zhang J, Li L, et al. Manual or electroacupuncture as an add-on therapy to SSRIs for depression: A randomized controlled trial. J Psychiatr Res 2019; 114: 24-33.

9. Kopp B, Lange $F$, Steinke A. The Reliability of the Wisconsin Card Sorting Test in Clinical Practice. Assessment 2021; 28(1): 248-263.

10. Siciliano M, Chiorri C, Battini V, Sant'Elia V, Altieri M, Trojano L, Santangelo G. Regression-based normative data and equivalent scores for Trail Making Test (TMT): an updated Italian normative study. Neurol Sci 2019; 40(3): 469-477.

11. Huang L, Liang YL, Hou JJ, Thai J, Huang YJ, Li JX, Zeng $Y$, Zhao XD. General Self-Efficacy Mediates the Effect of Family Socioeconomic Status on Critical Thinking in Chinese Medical Students. Front Psychol 2018; 9: 2578.

12. Montero-Marin J, Collado-Navarro C, Navarro-Gil M, Lopez-Montoyo A, Demarzo M, Herrera-Mercadal $P$, Barcelo-Soler A, Garcia-Campayo J. Attachment-based compassion therapy and adapted mindfulness-based stress reduction for the treatment of depressive, anxious and adjustment disorders in mental health settings: a randomised controlled clinical trial protocol. BMJ Open 2019; 9(10): e29909.

13. Kolahkaj B, Zargar F, Majdinasab N. The Effect of Mindfulness-Based Stress Reduction (MBSR) Therapy on Quality of Life in Women with Multiple Sclerosis, Ahvaz, Iran. J Caring Sci 2019; 8(4): 213-217. 
14. Andres-Rodriguez L, Borras X, Feliu-Soler A, PerezAranda A, Rozadilla-Sacanell A, Montero-Marin J, Maes $M$, Luciano JV. Immune-inflammatory pathways and clinical changes in fibromyalgia patients treated with Mindfulness-Based Stress Reduction (MBSR): A randomized, controlled clinical trial. Brain Behav Immun 2019; 80: 109-119.

15. Jalali $D$, Abdolazimi M, Alaei Z, Solati K. Effectiveness of mindfulness-based stress reduction program on quality of life in cardiovascular disease patients. Int $\mathrm{J}$ Cardiol Heart Vasc 2019; 23: 100356.
16. Prickett C, Brennan L, Stolwyk $R$. Examining the relationship between obesity and cognitive function: a systematic literature review. Obes Res Clin Pract 2015; 9(2): 93-113.

17. Abi CJ, Rammal S, Fares Y, Abou AL. Trail Making Test: normative data for the Lebanese adult population. Clin Neuropsychol 2020; 34(sup1): 29-42.

18. Min J, Zhou XX, Zhou F, Tan Y, Wang WD. A study on changes of the resting-state brain function network in patients with amnestic mild cognitive impairment. Braz J Med Biol Res 2019; 52(5): e8244. 